\title{
Tratamiento del papiloma oncocítico nasosinusal avanzado sin malignización asociada, con resección endoscópica y radioterapia en arcos de volumen modulado (RAVM). Reporte de un caso
}

\author{
Aderito De Sousa-Fontes ${ }^{1}$, Nelson Urdaneta-Lafée ${ }^{2,}$ Perfecto A. Abreu-Durán ${ }^{3}$, Héctor Rodríguez-Hernández ${ }^{4}$
}

\section{RESUMEN}

El papiloma oncocítico $(\mathrm{PO})$ es una neoplasia rara de la cavidad nasal y los senos paranasales y es el subtipo histológico menos frecuente del papiloma rinosinusal. Al igual que el papiloma invertido (PI), se lo considera un tumor benigno, pero su comportamiento biológico no lo es tanto por su crecimiento local agresivo, su tasa alta de recurrencia y el riesgo de transformación maligna con asociación a carcinoma. El tratamiento de elección es la resección endoscópica. Se ha usado exitosamente la radioterapia para el tratamiento de los PI extensos, pero no se conocen informes documentados de su uso en pacientes con PO avanzado o con extensión extrasinusal y sin malignización asociada. Se presenta un caso de PO unilateral izquierdo con enfermedad extensa que comprometía la pared nasal lateral y los senos maxilar, etmoidal y frontal del mismo lado, tratado con radioterapia en arcos de volumen modulado.

\section{PALABRAS CLAVE}

Cavidad Nasal; Papiloma Oncocítico; Radioterapia

1 Director, Unidad de Otorrinolaringoendoscopia, Instituto Médico La Floresta. Coordinador Fellow de Cirugía Endoscópica de Nariz, senos paranasales y Base de Cráneo. Profesor de la Unidad Interdisciplinaria de Cirugía Endoscópica de base de Cráneo. Profesor visitante del Posgrado de Neurocirugía, Hospital Militar Doctor Carlos Arvelo. Caracas, Venezuela.

2 Director, Unidad de Radioterapia Oncológica y Medicina Nuclear, Instituto Médico La Floresta. Profesor Visitante de Radioterapia, Smilow Cancer Hospital and Yale Cancer Center, Yale University (EUA). Exjefe, Cátedra de Radioterapia y Medicina Nuclear, Hospital Universitario, Universidad Central de Venezuela. Caracas, Venezuela.

3 Radioterapeuta adjunto de la Unidad de Radioterapia Oncológica (GURVE), Instituto Médico La Floresta. Caracas, Venezuela.

4 Físico médico, Unidad de Radioterapia Oncológica GURVE, Instituto Médico La Floresta. Caracas, Venezuela.

Correspondencia: Aderito De Sousa; aderitodesousa@gmail.com

Recibido: agosto 9 de 2016

Aceptado: octubre 14 de 2016

Cómo citar: De Sousa-Fontes A, Urdaneta-Lafée N, Abreu-Durán PA, Rodríguez-Hernández H. Tratamiento del papiloma oncocítico nasosinusal avanzado sin malignización asociada, con resección endoscópica y radioterapia en arcos de volumen modulado (RAVM). Reporte de un caso. latreia. 2017 0ct-Dic;30(4):448-454. D0I 10.17533/udea.iatreia.v30n4a09. 


\section{SUMMARY}

Management of advanced sinonasal non-malignant oncocytic papilloma with endoscopic resection and volumetric modulated arc radiotherapy (VMAR). Case report

Oncocytic papiloma (OP) is a rare neoplasm of the nasal cavity and paranasal sinuses. It is the least common histological subtype of sinonasal papilloma. $\mathrm{OP}$ is regarded as a benign tumor like inverted papilloma (IP), but its biological behavior is not so benign due to its aggressive local growth, the high rate of recurrences and the risk of malignant transformation with associated carcinoma. Endoscopic resection is the treatment of choice for OP. No relevant reports on the use of radiotherapy are known in patients with OP with advanced disease or extrasinonasal extension without associated malignancy, although its application in advanced IP has been successfull. We report a case of unilateral left OP with advanced disease that involved lateral nasal wall, maxillary, ethmoidal and frontal sinus on the same side, treated with volumetric modulated arc radiotherapy.

\section{KEYWORDS}

Nasal Cavity; Oncocytic Papilloma; Radiotherapy

\section{Resumo}

Gestão do Papiloma Oncocítico Nasossinusal avançado sem malignidade associada, com ressecção endoscópica e Radioterapia em Volume de Arcos de Modulado. Relato de caso

O Papiloma Oncocítico (PO) é uma neoplasia rara da cavidade nasal e os Seios Parinasais menos comum e representa o subtipo histológicos menos frequente dos Papilomas Nasossinusais (PNS). Assim como o Papiloma Invertido (PI), o PO é considerado um tumor benigno, mas o seu comportamento biológico não é tão beniogno devido a seu crescimento local agressivo, a sua elevada taxa de recorrência e o risco potencial de transformação maliogna com carcinoma associado.

A ressecção endoscópica é o tratamento de preferência do PO. Em pacientes com doença avançada ou extensão extrassinusal sem malignidade associada, não há relatórios relevantes do uso de radioterapia (RT), embora a sua aplicação no caso dos PI avançados, em que tem sido utilizado com sucesso. Um caso de PO unilateral é apresentado com doença extensa que comprometeu a parede lateral nasal e os seios maxilar, etmoidal e frontal do mesmo lado, que foi tratado com sucesso com cirurogia endoscópica e radioterapia em arco volumetricamente modulada (RAVM).

\section{PALAVRAS CHAVE}

Cavidade NasaI; Papiloma Oncocítico; Radioterapia

\section{INTRODUCCIÓN}

Los papilomas rinosinusales (PRS) son tumores benignos infrecuentes de comportamiento localmente agresivo, altamente recidivantes $y$ que pueden asociarse con malignización. Desde el punto de vista histopatológico se clasifican de la siguiente manera: papilomas invertidos o scheiredianos; papilomas fungiformes, evertidos o exofíticos y papilomas oncocíticos o de células cilíndricas.

A pesar de ser considerados neoplasias benignas, por no poseer la capacidad de dar metástasis, su comportamiento biológico agresivo les confiere ciertas características "sutiles de malignidad", debido a su predisposición a invadir estructuras adyacentes a la nariz y los senos paranasales, incluso en ausencia de malignidad histológica asociada.

El papiloma oncocítico (PO) o de células cilíndricas es el subtipo más infrecuente y constituye de $3 \%$ a $5 \%$ de los PRS. La resección quirúrgica es el tratamiento de elección en los PRS. En el papiloma invertido (PI) avanzado sin maliognidad histológica asociada, se ha utilizado excepcionalmente la radioterapia con buenos resultados. Sin embargo, no hay informes documentados sobre la aplicación de radioterapia en el tratamiento del PO avanzado y sin maliognización asociada.

\section{PRESENTACIÓN DEL CASO}

Hombre de 56 años de edad que consultó en febrero de 2015 por obstrucción nasal y cefalea, ambas de predominio izquierdo, \ secreción nasal espesa. EI 
estudio endoscópico de las cavidades nasales reveló una masa de aspecto polipoide vegetante en el corredor nasal izquierdo, con desviación homolateral del septum nasal.

La tomografía computada de los senos paranasales (TCSPN) reveló una opacificación siognificativa del corredor nasal y de los senos maxilar, etmoidal y frontal del lado izquierdo, con áreas de hiperostosis focal en la pared lateral y el seno frontal (figuras $1 \mathrm{~A} y 1 \mathrm{~B}$ ).

La resonancia magnética (RM) evidenció que este hallazoo se correspondía con una masa sólida, con un patrón contorneado de estrías hipointensas e hiperintensas alternas en su interior, sin signos de extensión extrasinusal (figuras 1C y 1D).

Se le practicaron maxilectomía medial y frontoetmoidectomía endoscópica, resecando numerosas lesiones de aspecto polipoide adosadas a la pared lateral nasal, que se extendían a los senos maxilar, etmoidal y frontal izquierdos, firmemente adheridas a la pared orbitaria, el receso frontal y el seno frontal, lo cual impidió la resección total.

El estudio histopatológico y la inmunohistoquímica de las lesiones resecadas fueron compatibles con el diagnóstico de PO, sin malignización asociada.

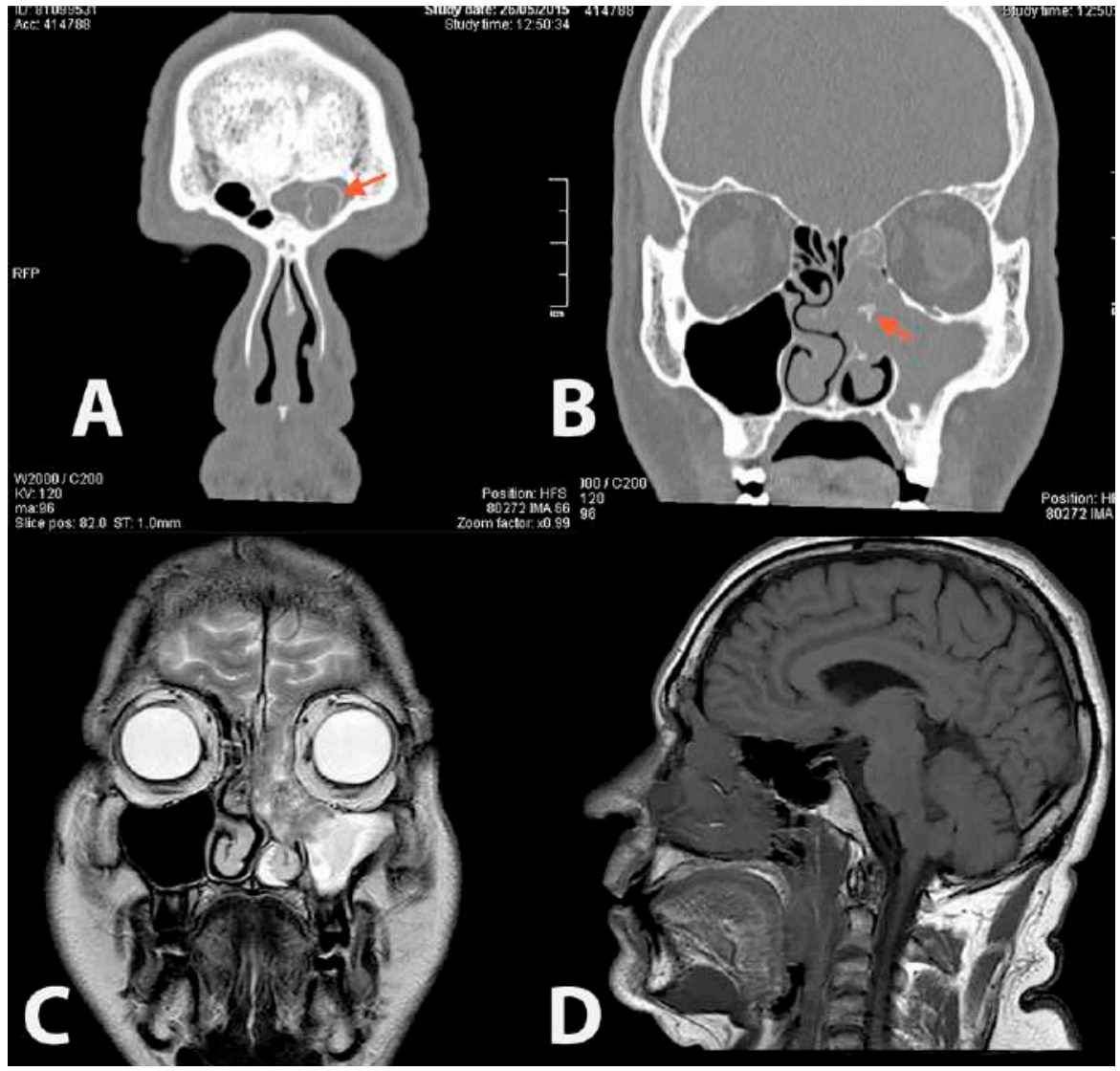

Figuras 1, A y B. Imágenes de TC coronal de senos paranasales, en las que se observa opacificación completa del corredor nasal izquierdo y los senos maxilar, etmoidal y frontal; la hiperostosis focal del hueso adyacente a la periferia de la neoplasia puede indicar el punto de origen del tumor 0 su adherencia estrecha a la pared ósea. C. Imagen de RM coronal en T2 sin contraste: se observa que el interior de la masa descrita presenta un patrón contorneado de áreas hiperintensas, separadas por estrías de baja intensidad (patrón cerebriforme), que se suele observar igualmente en los PI. D. Imagen de RM sagital en T1 sin contraste: se observa que la neoplasia se extiende desde el seno etmoidal hasta el frontal, sin afectar la cavidad craneal 
En el postoperatorio se sometió al paciente a un tratamiento adyuvante con radioterapia en arcos de volumen modulado (RAVM). Para la planificación de esta terapia, se elaboró previamente una máscara de inmovilización de la cabeza y el cuello con material termoplástico (Zentec ${ }^{T M}$, CIVCO Medical Solutions), se hizo un estudio de planificación con TC, utilizando la máscara de inmovilización y el protocolo de radioterapia (TC helicoidal con y sin contraste usando cortes de $1 \mathrm{~mm}$ de espesor), a fin de crear reconstrucciones tridimensionales de la anatomía del área afectada y de las zonas adyacentes comprometidas por la neoplasia. Adicionalmente, con la ayuda de una RM se hizo un registro rígido con las imágenes de TC de planificación, con la finalidad de fusionar las estructuras visibles con más detalle, delimitando el área correspondiente al volumen macroscópico del tumor (VMT), y los márgenes de enfermedad subclínica o volumen clínico del tumor (VCT) y el volumen de planificación del tumor (VPT), así como de las estructuras críticas vecinas de riesgo, como los nervios ópticos, el quiasma, el tallo cerebral, los ojos, las glándulas salivales y los oídos (figuras 2A y 2B).

El paciente recibió 4 arcos parciales de aplicación de radiación (dos axiales y dos sagitales). Luego de elegir los ángulos de inicio y finalización de cada arco, se procedió a la planificación inversa, utilizando los mismos criterios aplicados en la radioterapia de intensidad modulada. Esta tecnología permite administrar la terapia de manera precisa y en un tiempo sumamente corto (3 minutos) reduciendo sionificativamente los movimientos intrafracción (figura 3A, 3B, 3C y $3 \mathrm{D}$ ).

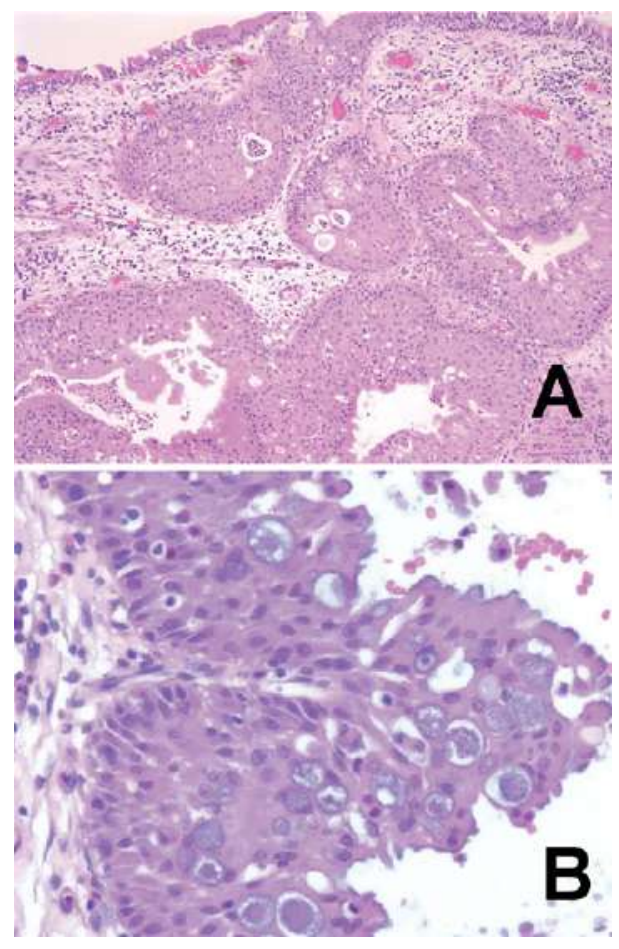

Figura 2. Cortes histológicos teñidos con hematoxilina-eosina con aumentos bajo (A) e intermedio (B). Se observa un crecimiento notable de células escamosas del epitelio respiratorio, con invaginaciones crípticas hacia el estroma y evaginaciones hacia la superficie epitelial (patrones exofítico y endofítico), dispuestas con un patrón de pseudoestratificación, características del PO. A mayor aumento (B) se detallan células vacuoladas con pequeños núcleos uniformes, oscuros y redondos y citoplasma eosinofílico. De igual manera, se pueden observar numerosos microquistes entre las células escamosas que contienen restos de células y mucina. La membrana basal, de densidad normal, se encuentra integra 


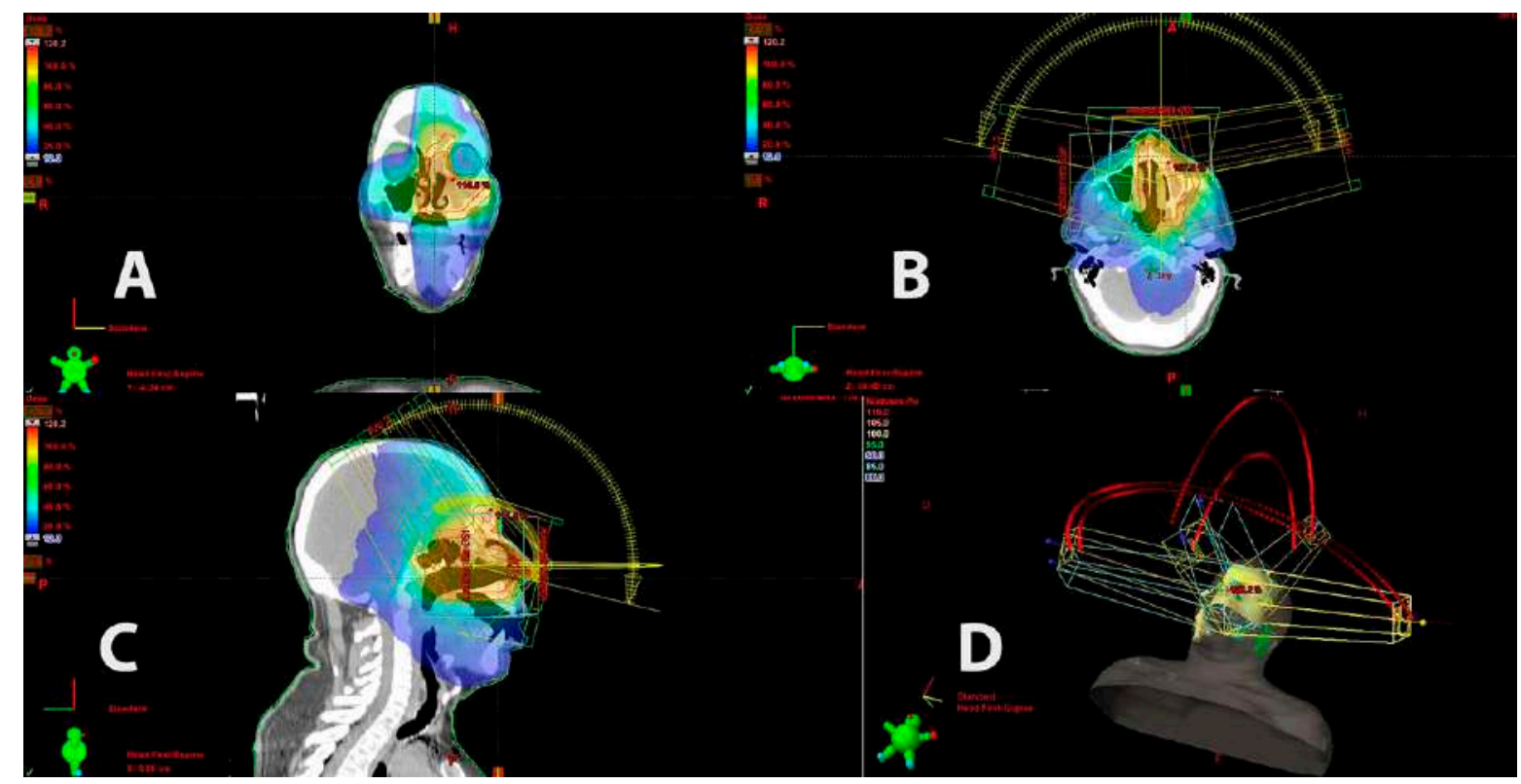

Figura 3: A: Corte coronal de la imagen de TC, la escala de colores que va desde el azul tenue hasta el rojo intenso representa la dosis impartida en el volumen siendo la zona roja el área afectada por la enfermedad y por lo tanto la zona de tratamiento con la mayor concentración de dosis. Se puede ver como la radioterapia de arcos volumétricos concentra la dosis en un área específica excluyendo zonas críticas cercanas como los ojos. B: Corte axial de la imagen de TC, en ella no solo se aprecia la degrades de la dosis impartida en el volumen, desde la zona afecta por la enfermedad (zona roja) hasta la zonas a excluir del tratamiento (zona azul), sino que vemos un representación esquemática de los arcos de tratamiento para este caso, se seleccionan segmentos circulares específicos para cada caso dependiendo de la ubicación y extensión de la enfermedad para asegurar que el haz modulado de tratamiento no pase por ninguna zona a riesgo. C: Corte sagital de la imagen de TC, la zona roja indica la zona de tratamiento cubierta por la isodosis prescrita 0 área de $100 \%$ de dosis y se aprecia que no necesariamente los arcos de tratamientos tienen que ser coplanares (que coexistan en el mismo plano) sino que se puede hacer usos de varios planos para ejecutar los arcos de tratamientos, de modo de optimizar la entrega de la dosis en área blanco y minimizar la dosis en las zonas a riesgo cercanas. D: Reconstrucción en 3D del cuerpo del paciente y su posición con respecto a los arcos de tratamientos, las líneas concéntricas de diferentes radios representan la cantidad de arcos ejecutados, por ejemplo si nos fijamos en plano sagital de la reconstrucción 3D observamos un par de líneas, esto representa dos arcos concéntricos uno en sentido horario y otro en sentido anti horario

La recuperación fue sin complicaciones y hasta el segundo año y 6 meses de seguimiento el paciente no ha presentado enfermedad recurrente.

\section{DISCUSIÓN}

EI papiloma fungiforme (PF), el papiloma invertido (PI) y el papiloma oncocítico (PO) son tres lesiones neoplásicas originadas en el epitelio nasosinusal con características histopatológicas distintas (1). Los PI y PO, a pesar de su histología diferente, tienen características clínicas similares: afectan la pared lateral nasal y los senos paranasales, con un elevado potencial de invasión local y cambios malignos, especialmente cuando son extensos $y$ se resecan de manera incompleta $(2,3)$.

La asociación con el carcinoma de células escamosas en el caso del PO es de aproximadamente $15 \%$ (4).

Barnes y colaboradores (5) señalaron que las células epiteliales del PO constituyen verdaderos oncocitos del epitelio respiratorio nasosinusal. Los oncocitos pueden observarse en neoplasias benignas $y$ malignas. EI aspecto oncocitoide del $\mathrm{pO}$ se debe al gran volumen que alcanzan estas células. Su eosinofilia granular citoplasmática se debe al gran 
contenido de mitocondrias, que histoquímicamente se correlaciona con el alto contenido de ATP-asa y enzimas oxidativas, por lo que también se las conoce como células oxifílicas.

EI PO es usualmente unilateral y se presenta por igual en ambos sexos, por lo general en la quinta y sexta décadas de la vida (6). La frecuencia de su asociación a malignidad es mayor (10\% a $17 \%$ ) que la del PI $(7,8)$.

Los hallazgos radiológicos del $\mathrm{PO}$ son similares a los del PI y varían según la evolución de la enfermedad: ocasionan opacificación de las cavidades nasosinusales afectadas y al progresar pueden erosionar las paredes óseas y desplazar las estructuras adyacentes $(8,9)$.

Histológicamente, el pO exhibe un patrón de crecimiento de células escamosas o transicionales del epitelio respiratorio exofítico y endofítico, dispuestas en un patrón de pseudoestratificación, con pequeños núcleos uniformes, oscuros y redondos, citoplasma eosinofílico y numerosos microquistes entre las células escamosas, que contienen restos de células y mucina $(6,8,9)$.

La resección endoscópica es, en la actualidad, el estándar de oro en el tratamiento del PO. Sin embargo, incluso después de haber sido resecado, puede recidivar, extenderse fuera de los límites nasosinusales y malionizarse.

Varios autores (10-16) han informado la eficacia de la RT en el tratamiento del PI biológicamente agresivo sin malignización asociada, especialmente cuando se utiliza como tratamiento adyurante después de la resección endoscópica, controlando el comportamiento agresivo de esta neoplasia y por ende reduciendo la recurrencia de la enfermedad y el riesgo de malignización. Sin embargo, la RT convencional está asociada con un alto riesgo de toxicidad $y$ efectos secundarios. Con las técnicas modernas como la radioterapia de intensidad modulada (RTIM) y la radioterapia guiada por imágenes (RTGI), es posible aumentar la dosis de radiación al volumen del tumor, reduciéndola en la periferia de la lesión y evitando la radiación de los órganos y estructuras limítrofes en situación de riesgo (ojos, nervio y quiasma ópticos, vasos importantes, tallo cerebral y el resto del SNC; además, preservando la función del órgano y mejorando la calidad de vida $(16,17)$.
No hay en la literatura médica informes sobre la aplicación de RT en el PO, pero el parentesco y muchas de las características afines de esta neoplasia con el PI hacen posible su utilidad potencial en el tratamiento del PO avanzado sin malignidad asociada, en tumores biológicamente agresivos con extensión extrasinusal, en enfermos con riesgo de morbilidad importante por los efectos de una cirugía radical, en casos con enfermedad resecada de manera incompleta $y$ en pacientes con recurrencia temprana.

\section{CONFLICTOS DE INTERESES}

Ninguno por declarar.

\section{REFERENCIAS BIBLIOGRÁFICAS}

1. Hyams VJ. Papillomas of the nasal cavity and paranasal sinuses. A clinicopathological study of 315 cases. Ann Otol Rhinol Laryngol. 1971 Apr;80(2):192-206.

2. Kaufman MR, Brandwein MS, Lawson W. Sinonasal papillomas: clinicopathologic review of 40 patients with inverted and oncocytic schneiderian papillomas. Laryngoscope. 2002 Aug;112(8 Pt 1):1372-7.

3. Yang $\mathrm{YJ}$, Abraham JL. Undifferentiated carcinoma arising in oncocytic Schneiderian (cylindrical cell) papiIloma. J Oral Maxillofac Surg. 1997 Mar;55(3):289-94.

4. Liu CY, Tsai TL, Hsu CY, Lin CZ. Oncocytic Schneiderian papilloma. J Chin Med Assoc. 2004 May;67(5):255-7.

5. Barnes L, Bedetti C. Oncocytic Schneiderian papilloma: a reappraisal of cylindrical cell papilloma of the sinonasal tract. Hum Pathol. 1984 Apr;15(4):344-51.

6. Yaprak N, Aşık M, Turhan M, Toru H. Özbudak İH, Oncocytic Schneiderian Papilloma. Akd Med J. 2015;2:113-6.

7. Maitra A, Baskin LB, Lee EL. Malignancies arising in oncocytic schneiderian papillomas: a report of $2 \mathrm{ca}-$ ses and review of the literature. Arch Pathol Lab Med. 2001 Oct;125(10):1365-7.

8. Karligkiotis A, Bignami M, Terranova P, Gallo S, Meloni F, Padoan G, et al. Oncocytic Schneiderian papillomas: Clinical behavior and outcomes of the endoscopic endonasal approach in 33 cases. Head Neck. 2014 May;36(5):624-30. DOI 10.1002/hed.23341. 
9. Cheng TY, Ueng SH, Chen YL, Chang KP, Chen TM. Oncocytic schneiderian papilloma found in a recurrent chronic paranasal sinusitis. Chang Gung Med J. 2006 May-Jun;29(3):336-41.

10. Suh KW, Facer GW, Devine KD, Weiland LH, Zujko RD. Inverting papilloma of the nose and paranasal sinuses. Laryngoscope. 1977 Jan;87(1):35-46.

11. Levendag PC, Annyas AA, Escajadillo JR, Elema JD. Radiotherapy for inverted papilloma: a case report. Radiother Oncol. 1984 Jun;2(1):13-7.

12. Hug EB, Wang CC, Montomery WW, Goodman ML. Management of inverted papilloma of the nasal cavity and paranasal sinuses: importance of radiation therapy. Int J Radiat Oncol Biol Phys. 1993 Apr;26(1):67-72.

13. Wright EJ, Chernichenko N, Ocal E, Moliterno J, Bulsara KR, Judson BL. Benign inverted papilloma with intracranial extension: prognostic factors and outcomes. Skull Base Rep. 2011 Nov;1(2):145-50. DOI 10.1055/s-0031-1287687.

14. Guedea F, Mendenhall WM, Parsons JT, Million RR. The role of radiation therapy in inverted papilloma of the nasal cavity and paranasal sinuses. Int J Radiat Oncol Biol Phys. 1991 Apr;20(4):777-80.

15. Gomez JA, Mendenhall WM, Tannehill SP, Stringer SP, Cassisi NJ. Radiation therapy in inverted papillomas of the nasal cavity and paranasal sinuses. Am J Otolaryngol. 2000 May-Jun;21(3):174-8.

16. Rutenberg M, Kirwan J, Morris CG, Werning JW, Mendenhall WM. Radiation therapy for sinonasal inverted papilloma. Pract Radiat Oncol. 2013 OctDec;3(4):275-81. DOI 10.1016/j.prro.2012.07.007.

17. Otto K. Volumetric modulated arc therapy: IMRT in a single gantry arc. Med Phys. 2008 Jan;35(1):310-7. 